\title{
Look-Angle-Constrained Control of Arrival Time with Exact Knowledge of Time-to-Go
}

\author{
Namhoon Cho ${ }^{*}$ and Seokwon Lee ${ }^{\dagger}$ \\ Cranfield University, Cranfield, Bedfordshire, MK43 OAL, United Kingdom
}

\section{Introduction}

The capability to control the time of arrival at a goal position as desired endows a single vehicle or a coalition of many of them with the strategic advantage to perform time-critical missions. Arrival time coordination can be used as an element to solve multi-agent, multi-depot routing and task planning problems in cooperative unmanned aerial robots. The tactic known as Salvo which either designates or synchronises the impact times across multiple missiles to enhance their collective survivability as well as attack effectiveness strongly depends on control of arrival time. In principle, control of arrival time is essentially adjustment of the arc length of the vehicle's flight path through manipulation of the curvature, provided that most vehicles flying in the atmosphere often prefer not to change their speeds excessively.

On the other hand, the capability to take measurements of the target with onboard sensors provides a higher degree of autonomy to the vehicle and hence allows a more intelligent behaviour. Modern autonomous vehicles acquire information about the designated destination or the surrounding environment with imaging sensors, in particular. An onboard sensor that collects emission or reflection from the target is usually not likely to be omni-directional yet possesses only a finite field-of-regard. The requirement to ensure continuous acquisition of target-originated signals necessitates a measure to keep the information source inside the sensor's field-of-view which spans over a solid angle of limited range. That is, a box constraint is imposed on the look angle.

Respecting the look angle box constraint significantly complicates the design of a manoeuvre policy when it is coupled with the necessity to control the arrival time. The demand to satisfy the desired flight time while obeying the look angle constraint drives an extensive amount of studies in the recent decade, particularly in the domain of missile guidance [1-14]. The approaches have been diversified by the choice of various control methodologies, controlled variables, and especially, the way of quantifying the time-to-go. The guidance methods proposed in [5-7, 9] are based on parametric shaping of range with respect to a function of look angle, parametrisation of range as a polynomial of time, nonlinear tracking of desired lead angle, and nonlinear regulation of the range and lead angle tracking errors, respectively. The time-to-go information in these methods are provided as their respective exact forms. However, their structures are not similar to the well-known classical guidance laws. On the other hand, the methods proposed in

\footnotetext{
*Research Fellow, Centre for Autonomous and Cyber-Physical Systems, School of Aerospace, Transport and Manufacturing, n.cho@cranfield.ac.uk, AIAA Member

†Research Fellow, Centre for Autonomous and Cyber-Physical Systems, School of Aerospace, Transport and Manufacturing, seokwon.lee@cranfield.ac.uk, AIAA Member
} 
[1, 10] are based on the proportional navigation, as it is preferable to exploit the widely-used legacy method to ensure operational reliability, particularly near the end of flight. However, the design and analysis in [1, 10] incorporate an approximate estimate for the time-to-go of the pure proportional navigation guidance law (PPNG) which arbitrarily assumes small lead angle. To complement the theoretical and numerical issues arising from the inaccurate time-to-go, another guidance method was proposed in [12] by using the exact time-to-go of PPNG derived in [15], but this method requires switching between policies. In summary, a method based on the PPNG with its exact time-to-go for arrival time control under look angle constraint is still absent despite a lot of previous efforts.

This study aims to develop a novel guidance synthesis framework for arrival time control under look angle box constraint with an elaborated formulation taking into consideration the exact knowledge of time-to-go for the baseline policy. To promote the generality of our development, the design framework is first developed considering the PPNG with a family of range-varying functions as the baseline policy. Then, a biased proportional navigation guidance law (BPNG) is derived by specifying the navigation ratio of the baseline PPNG to be a constant, the case for which a closed-form expression of the exact time-to-go is available. The proposed BPNG turns out to be a novel guidance law extending the earlier BPNGs in [10, 11] that are based on the approximate solution of the time-to-go for PPNG.

The following sections are organised to discuss the details of the new guidance method that leverages exact knowledge of the time-to-go for a baseline guidance law, namely, the pure proportional navigation guidance law. Section $\Pi$ presents the basic kinematic relations and formal description of the problem. The theory of the proposed guidance method is developed in Sec. [III where a new BPNG for control of arrival time under look angle restriction is derived as a special case of the generalised theory. Section IV presents numerical examples to illustrate the new BPNG with particular emphasis on the comparison with the existing guidance laws making use of approximate time-to-go expressions. The design lessons learnt are briefly discussed in Sec. $\mathrm{V}$

\section{Problem Formulation}

This section mathematically describes the problem considered in this study. The engagement kinematic equations are summarised, and the constraints that should be satisfied by the closed-loop trajectory are detailed.

Consider a planar engagement for which geometry can be represented in polar coordinates as shown in Fig. 1 . The figure depicts the situation where the vehicle $M$ flying at a speed of $V$ and exhibiting a lateral acceleration of $a$ moves toward the $\operatorname{target} T$. The angular variables are defined to be positive counter-clockwise. The variables $r, \lambda, \gamma$, and $\sigma$ denote range, line-of-sight angle, flight path angle, and lead angle, respectively. It is clear from the definition that $\sigma=\gamma-\lambda$.

The present study takes several assumptions for the sake of simplicity in further development. First, the target is assumed to be effectively a stationary point. Second, the vehicle is assumed to be lag-free in lateral acceleration and flying at a constant speed. Third, the look angle is supposed to be equivalent to the lead angle which is the angular 


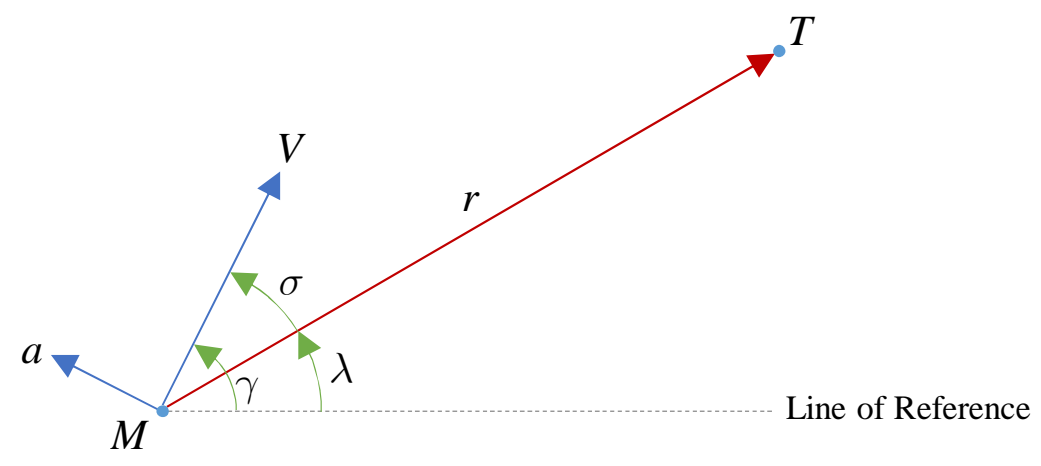

Fig. 1 Schematic Diagram of Planar Engagement with Definition of Variables

difference between the line-of-sight and the vehicle velocity. Fourth, the upper bound on the magnitude of look angle, which will be denoted from this point on by $\sigma_{\text {lim }}$, is less than $90 \mathrm{deg}$, implying that range $r$ decreases monotonically with respect to time $t$. With the assumptions stated above, the equations for the relative motion can be summarised as

$$
\begin{aligned}
\dot{r} & =-V \cos \sigma \\
\dot{\lambda} & =-\frac{V \sin \sigma}{r} \\
\dot{\gamma} & =\frac{a}{V} \\
\dot{\sigma} & =\frac{a}{V}+\frac{V \sin \sigma}{r}
\end{aligned}
$$

where the overdot notation stands for the differentiation with respect to time.

The free variable that is considered to be the input to the engagement kinematics described in Eqs. (1) through (4) is the lateral acceleration $a$. Let $t_{f_{d}}$ represents the desired arrival time given as a positive constant greater than or equal to $\frac{r_{0}}{V}$ where $r_{0}$ is the initial range. This study deals with the problem of finding a feedback control law, i.e., policy, that relates $a$ with other variables to satisfy the following requirements:

Requirement (1) Arrival at the desired time: $r \rightarrow 0$ as $t \rightarrow t_{f_{d}}$

Requirement (2) Boundedness of look angle: $|\sigma| \leq \sigma_{\lim }, \forall t \in\left[t_{0}, t_{f_{d}}\right]$

\section{Guidance Synthesis}

This section presents a novel guidance method that enables precise adjustment of arrival time as well as maintaining the goal position in the field-of-view spanning a symmetric interval around the line-of-sight. The augmentation approach that introduces an additional input term besides a given separate baseline input guaranteeing miss distance nullification is adopted to facilitate the design process so that the designer can focus only on satisfying the constraints imposed on arrival time and look angle with the additional input. In Sec. III.A a design framework in which the PPNG with a family 
of range-varying functions for the navigation ratio is taken as the baseline guidance law is proposed, and relevant design equations are presented considering the general expression for the exact time-to-go. The proposed method realises adjustment of arrival time through the design pursuing finite-horizon convergence of the predicted arrival time error. Then, a BPNG is newly proposed in Sec. III.B as a particular case of the proposed framework by setting the navigation ratio to be a constant.

\section{A. General Framework: Range-Varying Navigation Ratio}

\section{Trajectory and Time-to-Go Solutions for Baseline Guidance Law}

This study considers the PPNG as the baseline guidance law. The design process begins with investigating the trajectory solutions resulting from the baseline guidance law. Trajectory predictions are necessary at each instance to measure how close the predicted final state is from the desired final state.

The lateral acceleration commanded by the PPNG can be written as

$$
a_{\text {base }}=N(r) V \dot{\lambda}=-\frac{N(r) V^{2}}{r} \sin \sigma
$$

where $N(r)>1$ is a range-dependent navigation ratio. Because the range decreases monotonically under the assumption that the look angle is always an acute angle, the differential relation of the look angle with respect to the range can be obtained by dividing Eq. (4) with Eq. (1) as

$$
\frac{d \sigma}{d r}=\frac{\dot{\sigma}}{\dot{r}}=-\frac{\frac{a}{V}+\frac{V}{r} \sin \sigma}{V \cos \sigma}
$$

Substituting $a=a_{\text {base }}$ in Eq. (6) yields the range-derivative of the look angle under the baseline guidance law as

$$
\frac{d \sigma}{d r}=\frac{N(r)-1}{r} \tan \sigma
$$

Note that Eq. (7) is a separable differential equation which can be readily solved by integration. By rearranging Eq. (7) about each variable and then by integrating from the current time to a future time, we have

$$
\int_{\sigma}^{\tilde{\sigma}} \cot \sigma d \sigma=\int_{r}^{\tilde{r}} \frac{N(r)-1}{r} d r
$$

where $\tilde{r}$ and $\tilde{\sigma}$ are the range and look angle at the future time, respectively. Therefore, the look angle solution for the baseline guidance law can be obtained from Eq. (8) as

$$
\sin \tilde{\sigma}=\sin \sigma \exp \left\{\int_{r}^{\tilde{r}} \frac{N(x)-1}{x} d x\right\}
$$


Equation 9 indicates that the predicted look angle trajectory for the loop closed with the baseline guidance law is a function only of the range. One may notice from Eq. (1) that the differential relation between the range and the time under the same baseline policy can also be viewed as a separable differential equation since $\tilde{\sigma}=\tilde{\sigma}(\tilde{r})$. Rearranging Eq. (1) and integrating the result from the current time to the final time gives

$$
\int_{t}^{t_{f}} d t=-\frac{1}{V} \int_{r}^{r_{f}=0} \sec \tilde{\sigma}(s) d s
$$

Let $t_{g o} \triangleq t_{f}-t$ represents the time-to-go. Substitution of Eq. 97 into Eq. 10 results in an integral form expression for the time-to-go of the baseline guidance law.

$$
\begin{aligned}
t_{\text {go base }} & =\frac{1}{V} \int_{0}^{r} \sec \tilde{\sigma}(s) d s=\frac{1}{V} \int_{0}^{r} \frac{1}{\sqrt{1-\sin ^{2} \tilde{\sigma}(s)}} d s \\
& =\frac{1}{V} \int_{0}^{r} \frac{1}{\sqrt{1-\sin ^{2} \sigma \exp \left\{2 \int_{r}^{s} \frac{N(x)-1}{x} d x\right\}}} d s
\end{aligned}
$$

It can be noticed from Eq. (11) that the time-to-go is a function of both current range $r$ and current look angle $\sigma$, i.e., $t_{\text {go }}=t_{\text {gase }}(r, \sigma)$. For brevity of further writing, let $f(r, \sigma, s) \triangleq \frac{1}{\sqrt{1-\sin ^{2} \sigma \exp \left\{2 \int_{r}^{s} \frac{N(x)-1}{x} d x\right\}}}$ refers to the integrand function.

\section{Definition of Error and Derivation of Dynamics}

The arrival time control problem can be formulated into a terminal control of the relevant error variable. This study considers the arrival time error, or equivalently, the time-to-go error, to represent the design objective, which is defined with respect to the arrival time predicted at each instance with the baseline guidance law as

$$
e_{t}=\hat{t}_{f_{\text {base }}}-t_{f_{d}}=t_{\text {go base }}-t_{g o_{d}}
$$

where $t_{g o_{d}} \triangleq t_{f_{d}}-t$.

The baseline guidance law is responsible for delivering the vehicle to the target, but the arrival time depends only on the initial heading error when no other corrective action than the baseline is exerted. Hence, an additional input is necessary to enable adjustment of the arrival time. This study adopts the command augmentation approach introducing a bias command apart from the baseline command to use as the actuation to the arrival time error dynamics, that is, the lateral acceleration is given by

$$
a=a_{\text {base }}+a_{\text {bias }}=-\frac{N(r) V}{r} \sin \sigma+a_{\text {bias }}
$$

The form above is generally known as the BPNG. 
The time-derivative of the time-to-go solution will be necessary to design a controller that leverages feedback over the tracking error in time-to-go. To compute the time-derivative of the time-to-go solution, its partial derivatives with respect to the range and the look angle are needed. First, the partial derivatives of the integrand function with respect to the variables $r, s$, and $\sigma$ can also be obtained as follows:

$$
\begin{aligned}
& \frac{\partial}{\partial r} f(r, \sigma, s)=-\sin ^{2} \sigma \frac{N(r)-1}{r} f^{3}(r, \sigma, s) \exp \left\{2 \int_{r}^{s} \frac{N(x)-1}{x} d x\right\} \\
& \frac{\partial}{\partial s} f(r, \sigma, s)=\sin ^{2} \sigma \frac{N(s)-1}{s} f^{3}(r, \sigma, s) \exp \left\{2 \int_{r}^{s} \frac{N(x)-1}{x} d x\right\} \\
& \frac{\partial}{\partial \sigma} f(r, \sigma, s)=\sin \sigma \cos \sigma f^{3}(r, \sigma, s) \exp \left\{2 \int_{r}^{s} \frac{N(x)-1}{x} d x\right\}
\end{aligned}
$$

Equations (14)-(15) show that the partial derivatives of $f$ are related to each other as

$$
-\cot \sigma \frac{r}{N(r)-1} \frac{\partial}{\partial r} f(r, \sigma, s)=\cot \sigma \frac{s}{N(s)-1} \frac{\partial}{\partial s} f(r, \sigma, s)=\frac{\partial}{\partial \sigma} f(r, \sigma, s)
$$

Now, the partial derivatives of the integral form in Eq. (11) can be obtained by using the Leibniz integral rule as follows:

$$
\begin{aligned}
& \frac{\partial t_{\text {go } o_{\text {base }}}}{\partial r}=\frac{1}{V} \sec \sigma+\frac{1}{V} \int_{0}^{r} \frac{\partial}{\partial r} f(r, \sigma, s) d s \\
& \frac{\partial t_{\text {go } o_{\text {base }}}}{\partial \sigma}=\frac{1}{V} \int_{0}^{r} \frac{\partial}{\partial \sigma} f(r, \sigma, s) d s
\end{aligned}
$$

Finally, the dynamics of the error in the predicted arrival time can be derived by differentiating Eq. (12) with respect to time and using Eqs. (1), (4), (13), (17)-(19) as follows:

$$
\begin{aligned}
& \dot{e}_{t}=\dot{t}_{\text {go }_{\text {base }}}+1=\dot{r} \frac{\partial t_{\text {go }_{\text {base }}}}{\partial r}+\dot{\sigma} \frac{\partial t_{\text {go }_{\text {base }}}}{\partial \sigma}+1 \\
& =-\cos \sigma\left(\sec \sigma+\int_{0}^{r} \frac{\partial}{\partial r} f(r, \sigma, s) d s\right)+\left(\frac{a_{\text {bias }}}{V^{2}}-\frac{N(r)-1}{r} \sin \sigma\right) \int_{0}^{r} \frac{\partial}{\partial \sigma} f(r, \sigma, s) d s+1 \\
& =b(r, \sigma) a_{\text {bias }}
\end{aligned}
$$

where

$$
b(r, \sigma) \triangleq \frac{1}{V^{2}} \int_{0}^{r} \frac{\partial}{\partial \sigma} f(r, \sigma, s) d s
$$

One may notice from Eq. 20, that the arrival time error dynamics is exactly linear with respect to the bias command $a_{\text {bias. }}$. Note from Eq. (16) that $b(r, 0)=0, \operatorname{sign}(b(r, \sigma))=\operatorname{sign}(\sigma)$, and $|b(r, \sigma)|=|b(r,-\sigma)|$ for every $r \geq 0$.

Also, the BPNG defined Eq. (13) renders the dynamics of the look angle as

$$
\dot{\sigma}=\frac{a_{\text {bias }}}{V}-\frac{N(r)-1}{r} V \sin \sigma
$$


In summary, the differential equations for the arrival time error and the look angle in Eqs. 20, and 22, respectively, together describe the system dynamics that lays foundation for the design and analysis of a bias command $a_{\text {bias }}$.

\section{Design and Analysis of Guidance Laws}

The trajectory resulting from the guidance law should verify the following conditions to meet the requirements aforementioned in Sec. II.

Condition (1) $e_{t} \rightarrow 0$ as $t \rightarrow t_{f_{d}}$

Condition (2) $\frac{d}{d t}\left(\frac{1}{2}|\sigma|^{2}\right)=\sigma \dot{\sigma}=\sigma\left(\frac{a_{\text {bias }}}{V}-\frac{N(r)-1}{r} V \sin \sigma\right)<0$ at $\sigma= \pm \sigma_{\lim }$

If Condition (2) is satisfied by the closed-loop system, the magnitude of look angle will not exceed the given limit.

To achieve the goals, this study proposes a linear feedback policy for the bias command as

$$
a_{\text {bias }}=-k(\cdot) \phi(\sigma) \operatorname{sign}(\sigma) e_{t}
$$

where $k(\cdot)$ is the function representing the variable feedback gain and $\phi(\sigma)$ is the activation function, with the nonzero sign function defined by

$$
\operatorname{sign}(x) \triangleq \begin{cases}1 & \text { if } x \geq 0 \\ -1 & \text { if } x<0\end{cases}
$$

The designer-defined gain shaping functions satisfy the following conditions.

$$
\begin{gathered}
k(\cdot)>0 \\
0 \leq \phi(\sigma)=\phi(-\sigma) \leq 1, \forall \sigma \in\left[-\sigma_{\lim }, \sigma_{\lim }\right] \\
\phi\left( \pm \sigma_{\lim }\right)=0, \phi(0)=1
\end{gathered}
$$

The properties of the proposed BPNG in view of the design requirements should be discussed. To this end, the closed-loop systems dynamics is obtained by substituting Eq. (23) into Eqs. (20) and (22) as

$$
\begin{aligned}
\dot{e}_{t} & =-|b(r, \sigma)| k(\cdot) \phi(\sigma) e_{t} \\
\dot{\sigma} & =-\frac{k(\cdot) \phi(\sigma) \operatorname{sign}(\sigma) e_{t}}{V}-\frac{N(r)-1}{r} V \sin \sigma
\end{aligned}
$$

Note from Eq. 28) that $\dot{e}_{t}$ vanishes at $\sigma=0$ regardless of the value of $e_{t}$ for the given linear-in-error bias command since the gain $k$ is strictly positive by definition. Nonetheless, if $\sigma=0$ while $e_{t} \neq 0$, we have $\dot{\sigma} \neq 0$ from Eq. 29, hence, the closed-loop trajectory will not stay identically on the line $\sigma(t) \equiv 0$, and consequentially, $\dot{e}_{t}$ will be nonzero as soon as $\sigma$ becomes nonzero. 
Consider the Lyapunov function $\mathcal{V}=\frac{1}{2} e_{t}^{2}$ for the analysis of arrival time error convergence. It is clear from Eq. (28) that the time-derivative of the Lyapunov function satisfies

$$
\dot{\mathcal{V}}=-2|b(r, \sigma)| k(\cdot) \phi(\sigma) \mathcal{V} \leq 0
$$

The gain function $k$ attains a positive value as defined in Eq. (25). Thus, Eq. (30) shows that $\mathcal{V}$ monotonically decreases toward zero as long as $\sigma \neq 0, \pm \sigma_{\lim }$. It should be emphasised that the negative semi-definiteness of $\mathcal{V}$ alone does not automatically guarantee satisfaction of Condition (1). A proper gain function is necessary to produce enough rate of convergence in the time response of $e_{t}$ since its convergence to zero should take place well ahead of the desired arrival time to really achieve the given requirement. A simple choice for the gain function which is well-defined except at the point $r=0$ is

$$
k(t)=\frac{K V^{2}}{t_{\text {go base }}}
$$

where $K$ is a positive constant. The designer can shape the response characteristics through tuning of $K$. This particular example function is to enforce growth in the feedback gain as $r$ decreases to 0 . Note that the proposed design for the bias command avoids leveraging inversion of the control effectiveness function in the error dynamics, i.e., $b(r, \sigma)$, in order to specify the closed-loop error dynamics by a desired form. Although the method of feedback linearisation may facilitate the stability analysis and provide more explicit understanding of the error response, this deliberate decision to leave $b(r, \sigma)$ in the resulting closed-loop dynamics is to avoid any singularity in the bias command near $\sigma=0$ due to $b^{-1}(r, \sigma)$ becoming unbounded. More rigorous and complete analysis of the settling time in the convergence of $e_{t}$ without incurring any potential singularity in the bias command remains an open issue.

The look angle is always confined within the permissible region as long as the initial look angle is less than or equal to the limit value in its magnitude, since the activation function nullifies the bias command at either sides of the boundary. Considering the property in Eq. 27 and the fact that $N(r)>1$ by definition, the time-derivative of $\frac{1}{2}|\sigma|^{2}$ evaluated at $\sigma= \pm \sigma_{\lim }$ turns out to satisfy Condition (2), that is,

$$
\left.\frac{d}{d t}\left(\frac{1}{2}|\sigma|^{2}\right)\right|_{\sigma= \pm \sigma_{\lim }}=-\frac{N(r)-1}{r} V \sigma_{\lim } \sin \sigma_{\lim }<0
$$

Thus, if $\left|\sigma_{0}\right| \leq \sigma_{\text {lim }}$, then the proposed guidance law guarantees that $|\sigma|<\sigma_{\lim }, \forall t \geq t_{0}$.

\section{B. Special Case: Constant Navigation Ratio}

Specifying the baseline policy in the generalised synthesis framework by the PPNG with a constant navigation ratio yields a new BPNG that can meet the constraints described in Sec. II. The PPNG has many valuable properties supporting the strong preference given to it in design practices including stability in the homing phase and simplicity in 
implementation. Yet, in the present context, the PPNG with a constant navigation ratio poses itself as a favourable choice for the baseline policy in that closed-form expressions are available for the exact solutions of the closed-loop trajectory and time-to-go. In particular, a closed-form expression for the time-to-go enables easy evaluation of the arrival time error that enters into the command equation.

Suppose that $N(r)=N>1$ is now a constant. The expression for the time-to-go of PPNG can be rewritten in this case as

$$
t_{\text {go } o_{\text {base }}}=\frac{1}{V} \int_{0}^{r} \frac{1}{\sqrt{1-\sin ^{2} \sigma\left(\frac{s}{r}\right)^{2(N-1)}}} d s=\frac{r}{V} \frac{|\sin \sigma|^{-\frac{1}{N-1}}}{2(N-1)} B\left(\sin ^{2} \sigma ; \frac{1}{2(N-1)}, \frac{1}{2}\right)
$$

where $B(x ; a, b) \triangleq \int_{0}^{x} t^{a-1}(1-t)^{b-1} d t$ is the incomplete beta function. Also, a simple formula for the control effectiveness function $b(r, \sigma)$ can be derived by simplifying the integral expression. By applying the relation of Eq. (17) in Eq. 21] and then by integrating by parts, we obtain

$$
\begin{aligned}
& b(r, \sigma)=\frac{\cot \sigma}{(N-1) V^{2}} \int_{0}^{r} s \frac{\partial}{\partial s} f(r, \sigma, s) d s=\frac{\cot \sigma}{(N-1) V^{2}}\left\{r f(r, \sigma, r)-\int_{0}^{r} f(r, \sigma, s) d s\right\} \\
& =\frac{\cot \sigma\left(r \sec \sigma-V t_{\text {go }} \text { base }\right)}{(N-1) V^{2}}
\end{aligned}
$$

\section{Numerical Simulation}

Numerical simulation is performed to demonstrate response characteristics and performance benefits of the proposed guidance law. The simulation aims to show the effect of using an exact solution for the time-to-go through comparison with an existing method developed based on approximate solution for the time-to-go in [10]. The bias command presented in the previous study can be written as

$$
a_{\text {bias }}=-\frac{C V^{2}(2 N-1)}{\hat{t}_{\text {go }_{\text {base }}} r \sin ^{2} \sigma} \phi(\sigma) e_{t} \text { with } \hat{t}_{\text {go base }}=\frac{r}{V}\left\{1+\frac{\sin ^{2} \sigma}{2(2 N-1)}\right\}
$$

where $C$ is a constant gain. For implementing both the proposed method and the existing method, the navigation ratio entering into the baseline PPNG command is considered to be a constant. A fixed-step solver with the time-step of $10^{-4} \mathrm{~s}$ is used for numerical integration. Also, a command limiter is employed to restrict the magnitude of acceleration command below $100 \mathrm{~m} / \mathrm{s}^{2}$. The simplest point mass kinematics is used as the simulation model, and all signals fed into the guidance law are not corrupted by any source of imperfection such as noise or transport delay. Simulation parameters used are summarised in Table 1 The initial condition, the design parameters $C, N$, and the gain shaping function $\phi(\sigma)$ are chosen to be the same as the combination considered in [10] to clearly show the benefit of the proposed method while being consistent with the previous work. Interested readers may refer to [10] for more detailed discussions about the choice of the tunable elements of the method. 


\begin{tabular}{ccc}
\hline Parameter & Unit & Value \\
\hline$K$ & - & 0.03 \\
$C$ & - & 8 \\
$N$ & - & 3 \\
$\phi(\sigma)$ & - & $\cos \left(\frac{\pi}{2}\left(\frac{\sigma}{\sigma_{\text {lim }}}\right)^{5}\right)$ \\
$\sigma_{\text {lim }}$ & deg & 50 \\
$t_{f_{d}}$ & $\mathrm{~s}$ & 40 \\
$\left(X_{M_{0}}, Y_{M_{0}}\right)$ & $\mathrm{m}$ & $(0,0)$ \\
$V$ & $\mathrm{~m} / \mathrm{s}$ & 300 \\
$\gamma_{0}$ & deg & 30 \\
$\left(X_{T_{0}}, Y_{T_{0}}\right)$ & $\mathrm{m}$ & $(10000,0)$ \\
\hline
\end{tabular}

Table 1 Simulation Parameters: Design Parameters and Initial Condition

Figure 2 shows the simulation results and Fig. 3 provides a magnified view over the final second. The vehicle keeps closing to the target and eventually arrives at the goal point after the desired flight time (See Figs. 2a and 2c). The look angle does not violate the box constraint during the entire flight as shown in Fig. $2 \mathrm{~b}$. Overall performance of both guidance laws are similar in that the two guidance laws leverage regulation of the arrival time error in order to satisfy the desired arrival time constraint. Also, both methods produce smooth acceleration commands, preventing abrupt and irregular responses. However, the proposed guidance law shows more effective and desirable behaviour in comparison to the existing guidance law. The acceleration response of the existing method becomes highly unstable in the vicinity of the target, which may result in significant degradation of the performance in practice. In contrast, the vehicle employing the proposed method exhibits zero terminal acceleration as shown in Fig. 2f] since the baseline PPNG governs the overall response after the error is nullified. The arrival time error is kept small in the terminal phase as shown in Fig. 2d. nonetheless, even the error of a small magnitude can trigger the onset of instability in the acceleration response of the existing method. The highly oscillatory behaviour is also obvious in the bias command, the arrival time error, and the look angle for the existing method in Fig. 3 showing a more detailed look over the final second. The instability is thought to be a consequence of the singularity in the command that renders the command highly sensitive near zero look angle. Specifically, the inaccuracy in the time-to-go estimate used in the guidance command gives rise to the mismatch between the true error dynamics and the nominal error dynamics considered in the design process based on feedback linearisation. As a result, the term $e_{t}$ in the numerator becomes small but non-zero at the desired final time while the term $r \hat{t}_{g o_{\text {base }}} \sin ^{2} \sigma$ in the denominator approaches faster to zero. Also, the proposed method shows faster rate of error convergence as well as less control effort as shown in Figs. 2d and 2e. The results indicate that the design based on the exact time-to-go information can improve the response characteristics as well as the performance in terms of achieved accuracy. 


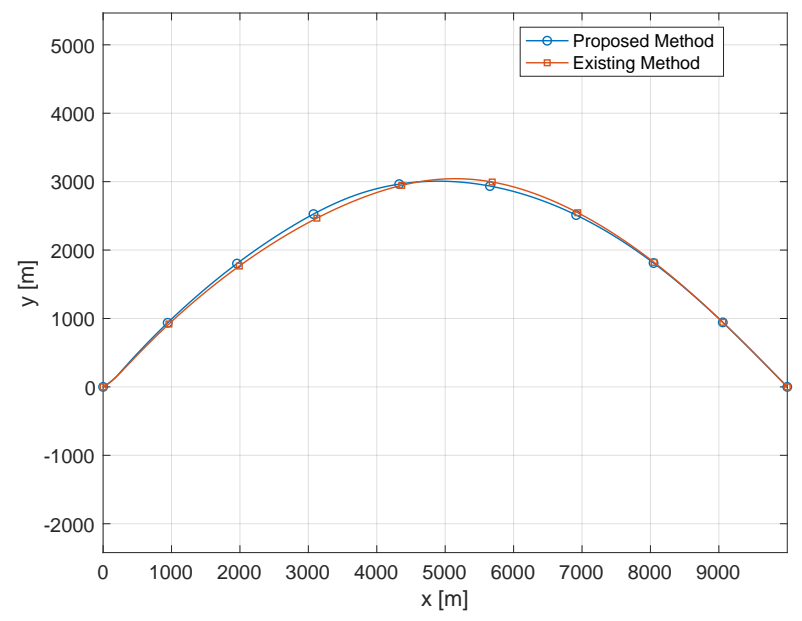

(a) Trajectories

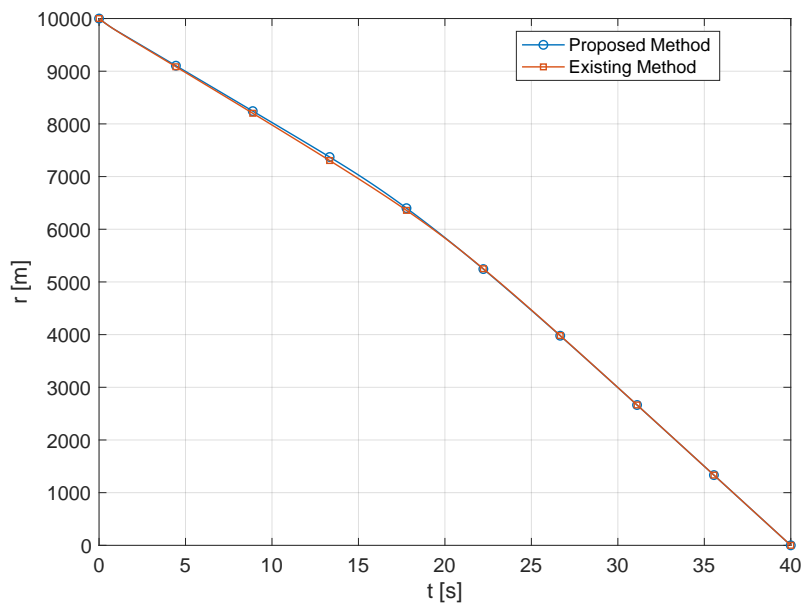

(c) Range

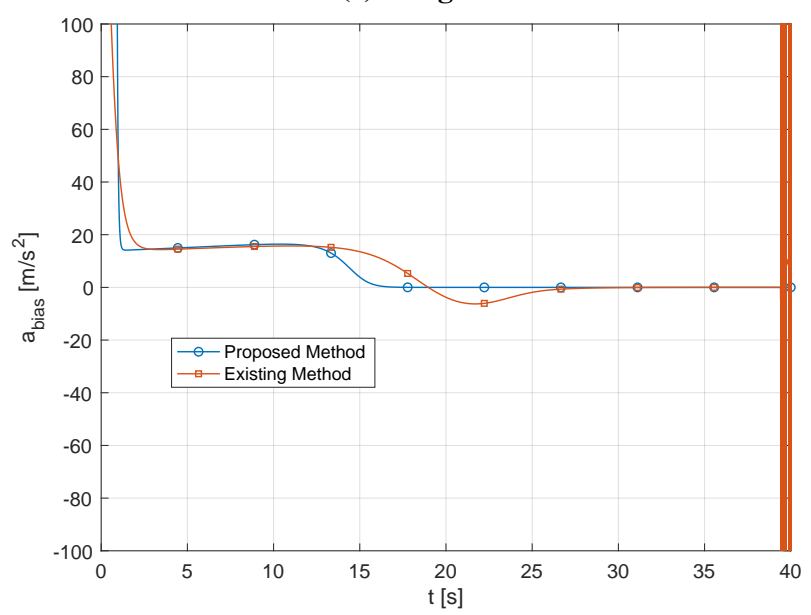

(e) Bias Command

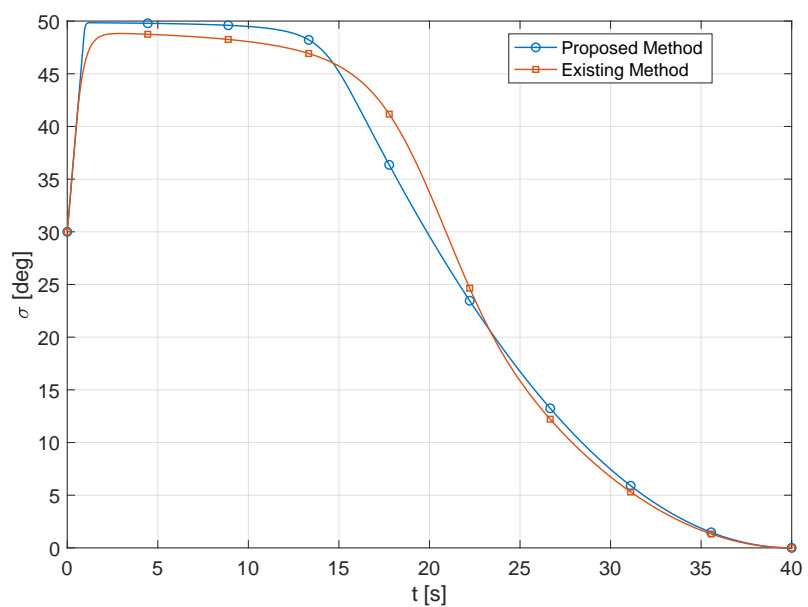

(b) Look Angle

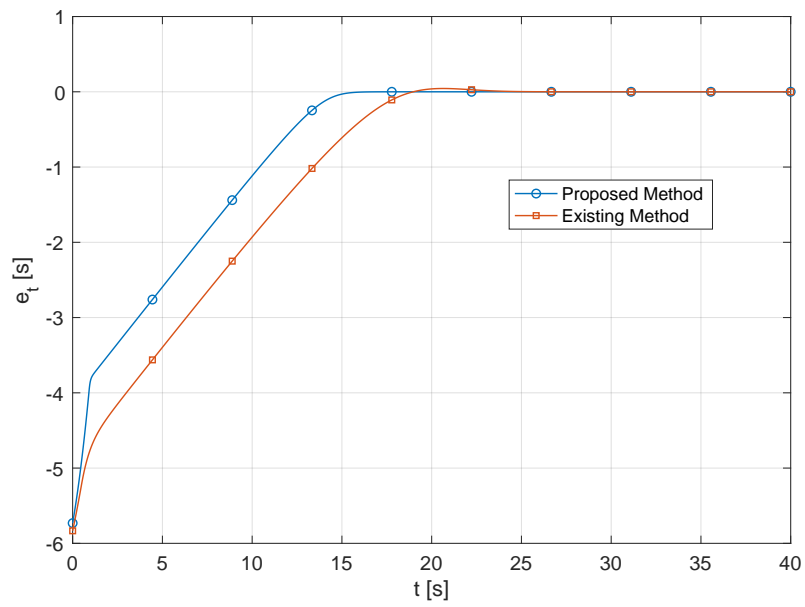

(d) Arrival Time Error

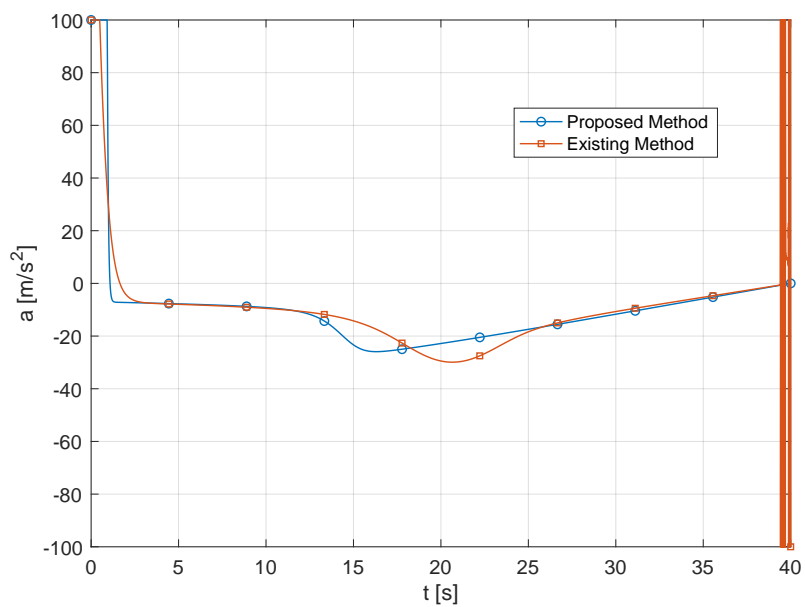

(f) Lateral Acceleration

Fig. 2 Simulation Results: Comparison with An Existing Guidance Law Based on Approximate Time-to-Go 


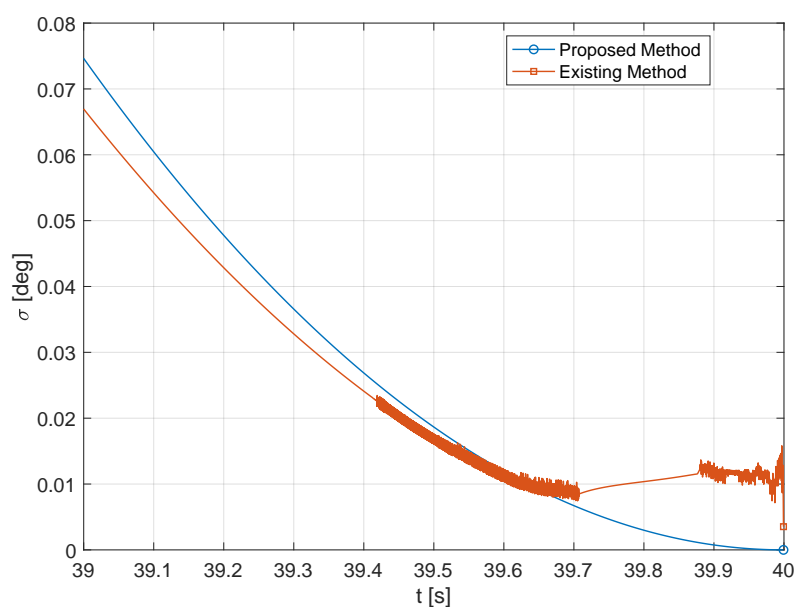

(a) Look Angle

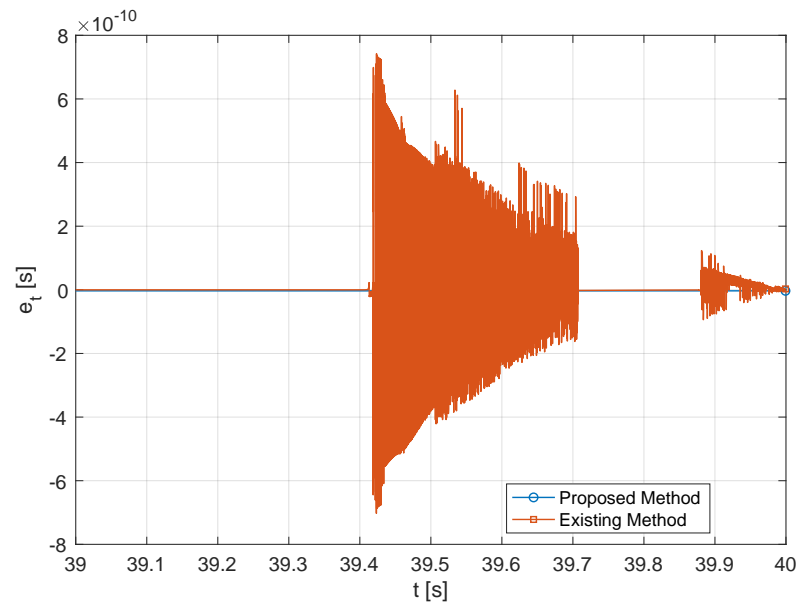

(c) Arrival Time Error

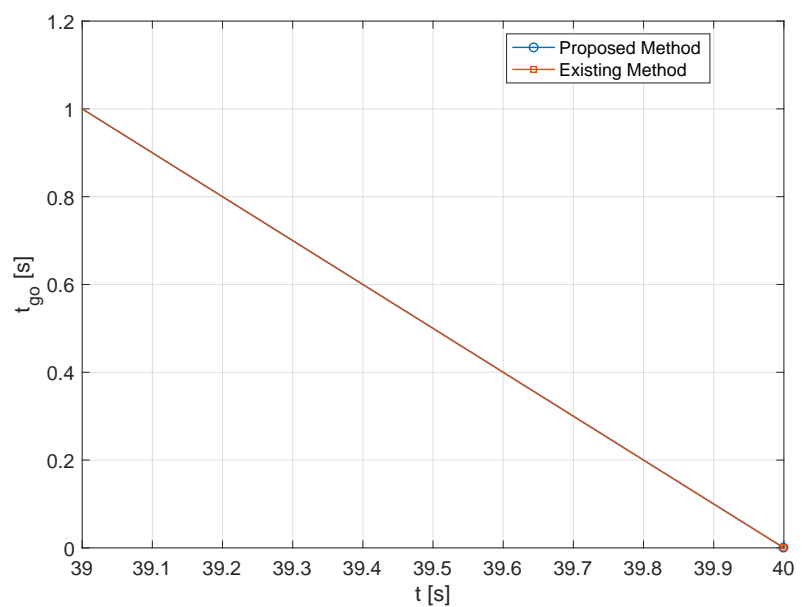

(b) Time-to-Go Estimate

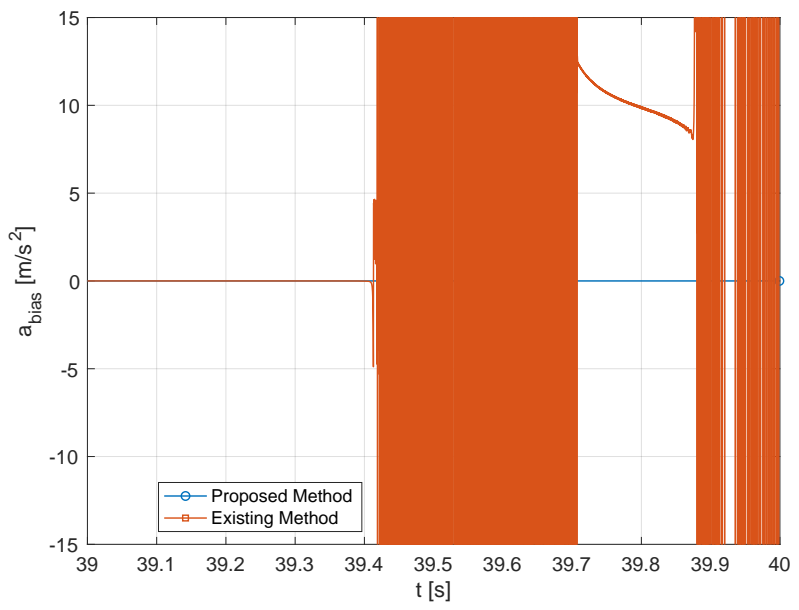

(d) Bias Command

Fig. 3 Simulation Results: Magnified View over the Final Second

\section{Conclusion}

In this study, a new biased proportional navigation guidance law was proposed as a solution to the arrival time control problem in the presence of the look angle inequality constraint. The main innovation in the proposed guidance law lies in the application of the exact expression for the time-to-go of baseline pure proportional navigation guidance law that enables accurate definition of the actual arrival time error. The results of analysis and numerical simulation point to a useful design lesson. If the linear-in-error feedback policy utilises a variable gain containing the reciprocal of time-to-go with the intention to force the hard final error constraint, it is better to utilise the exact solution for the time-to-go entering into the command to avoid command being unbounded near the desired final time. In conclusion, this study indicated the importance and the benefits of having an exact expression for time-to-go in the problem of arrival time control. The proposed method can readily be extended to a new distributed guidance method for simultaneous arrival of multiple vehicles through the application of a consensus protocol operating over the times-to-go. 


\section{References}

[1] Zhang, Y., Wang, X., and Wu, H., "Impact Time Control Guidance Law With Field of View Constraint," Aerospace Science and Technology, Vol. 39, 2014, pp. 361-369. doi:10.1016/j.ast.2014.10.002

[2] Zhang, Y., Wang, X., and Wu, H., "Impact Time Control Guidance with Field-of-View Constraint Accounting for Uncertain System Lag," Proceedings of the Institution of Mechanical Engineers, Part G: Journal of Aerospace Engineering, Vol. 230, No. 3, 2016, pp. 515-529. doi:10.1177/0954410015594401.

[3] Yang, Z., Lin, D., and Zang, L., "Impact Time Control Guidance Law Considering Seeker's Field-of-View Constraint Without Time-to-Go Information,” Journal of Advanced Computational Intelligence and Intelligent Informatics, Vol. 20, No. 3, 2016, pp. 412-417. doi:10.20965/jaciii.2016.p0412

[4] Kim, H.-G., and Kim, H. J., "Impact Time Control Guidance Considering Seeker's Field-of-View Limits,” IEEE Conference on Decision and Control, Las Vegas, NV, USA, 2016. doi:10.1109/CDC.2016.7798900

[5] Jeon, I.-S., and Lee, J.-I., "Impact-Time-Control Guidance Law With Constraints on Seeker Look Angle," IEEE Transactions on Aerospace and Electronic Systems, Vol. 53, No. 5, 2017, pp. 2621-2627. doi:10.1109/TAES.2017.2698837.

[6] Chen, X., and Wang, J., “Nonsingular Sliding-Mode Control for Field-of-View Constrained Impact Time Guidance,” Journal of Guidance, Control, and Dynamics, Vol. 41, No. 5, 2018, pp. 1214-1222. doi:10.2514/1.G003146.

[7] Tekin, R., Erer, K. S., and Holzapfel, F., “Impact Time Control with Generalized-Polynomial Range Formulation,” Journal of Guidance, Control, and Dynamics, Vol. 41, No. 5, 2018, pp. 1190-1195. doi:10.2514/1.G003279.

[8] Hu, J., Cui, N., Bai, Y., and Geng, Y., “Guidance Law to Control Impact Time Constraining the Seeker's Field of View?" Aircraft Engineering and Aerospace Technology, Vol. 91, No. 1, 2018, pp. 20-29. doi:10.1108/AEAT-06-2017-0151

[9] Kim, H.-G., and Kim, H. J., "Backstepping-Based Impact Time Control Guidance Law for Missiles With Reduced Seeker Field-of-View," IEEE Transactions on Aerospace and Electronic Systems, Vol. 55, No. 1, 2019 , pp. 82-94. doi:10.1109/TAES.2018.2848319

[10] He, S., Lee, C.-H., Shin, H.-S., and Tsourdos, A., “Optimal Three-Dimensional Impact Time Guidance with Seeker's Field-of-View Constraint," Chinese Journal of Aeronautics, Vol. 34, No. 2, 2021, pp. 240-251. doi:10.1016/j.cja.2020.04.006

[11] He, S., Lee, C.-H., Shin, H.-S., and Tsourdos, A., Optimal Guidance and Its Applications in Missiles and UAVs, Springer, Cham, Switzerland, 2020, Chap. 3. doi:10.1007/978-3-030-47348-8

[12] Lee, S., Cho, N., and Kim, Y., "Impact-Time-Control Strategy with a Composite Structure Considering the Seeker's Field-of-View Constraint," Journal of Guidance, Control, and Dynamics, Vol. 43, No. 8, 2020, pp. 1566-1574. doi:10.2514/1.G005063

[13] Mukherjee, D., and Kumar, S. R., "Nonlinear Impact Time Guidance with Constrained Field-of-View," American Control Conference, Denver, CO, USA, 2020. doi:10.23919/ACC45564.2020.9147958 
[14] Hong, H., Tekin, R., and Holzapfel, F., "Guaranteed Smooth Trajectory Generation for Field-of-View Constrained Impact-Time Control," Journal of Guidance, Control, and Dynamics, Vol. Online Published, 2021, pp. 1-7. doi:10.2514/1.G005723.

[15] Cho, N., and Kim, Y., "Modified Pure Proportional Navigation Guidance Law for Impact Time Control," Journal of Guidance, Control, and Dynamics, Vol. 39, No. 4, 2016, pp. 852-872. doi:10.2514/1.G001618 
$2021-05-26$

\section{Look-angle-constrained control of arrival time with exact knowledge of time-to-go}

Cho, Namhoon

AIAA

Cho N, Lee S. (2021) Look-angle-constrained control of arrival time with exact knowledge of time-to-go. Journal of Guidance, Control, and Dynamics, Volume 44, Number 10, October 2021 , pp. 1902-1908

https://doi.org/10.2514/1.G006000

Downloaded from Cranfield Library Services E-Repository 